\title{
Editorial
}

\section{Are we really in safe hands? How safe is safe?}

\author{
Pradhan R, Shrestha K, Gurung S \\ BP Koirala Institute of Health Science, Dharan, Nepal.
}

$\mathrm{D}$ ental workplaces can be potential sites for the spread of various communicable -- and incurable - diseases; to, from and among patients. Such diseases include viral hepatitis, herpes, syphilis, gonorrhea, tuberculosis and AIDS (Acquired Immunodeficiency Syndrome) to name a few. Are we dentists really doing justice to our patients and profession or are we unwitting vectors of disease?

Oral and dental diseases have become a major public health concern in both developed and developing countries. As one of the least developed countries in South Asia, Nepal must struggle to have adequate financial or human resources and the capacity to manage the epidemics of the infectious and communicable diseases. Dental surgeons have to work in a pathogen-rich, often blood, and contaminated environment. Because they are exposed to a variety of microorganisms present in blood and saliva, coupled with possible injury from the sharp instruments while treating the patients, doctors themselves become susceptible to different infectious diseases. To take HIV (Human immunodeficiency virus) as an example, we are expected to treat every single patient, some of whom may be infected with HIV and may be infectious. Thus all concerned must assess the current state of dental safety and analyze it. Oral health care workers must be equipped to protect themselves, their patients, and the community from contracting and spreading infectious diseases.

Patients can be infected by health care workers as well. Transmission of HIV to six patients of a dentist with AIDS has been reported by American Internal Medicine in 1992. Reports published from 1970 through 1987 document nine clusters HBV (Hepatitis B virus) in which patients were infected by treatment from an infected dental health care worker.

There are effective infection control procedures and universal precautions for dental clinics and dental operatories to prevent cross contamination, which should be practiced by dentists, dental technicians, dental chair-side assistants, and dental lab technicians. However, this issue has not been adequately studied. As a first step, we conducted a descriptive, cross sectional survey of 132 dental surgeons to assess the current practice of infectious disease control in private dental clinics in the Kathmandu valley.

Sterilization equipment: According to the popular publications, autoclave remains the preferred method of sterilization. The advantage of autoclaves is the efficient destruction of most of the resistant bacteria spores in brief intervals of exposure, the easy control of quality and lethality, and the absence of toxic residue on materials following the sterilization process. The present study showed that less than half of dentists in the sample had autoclave as their sterilizing instrument.

Although $97 \%$ of the dental clinics in the study had some sterilization instruments, the presence of equipment doesn't guarantee good sterilization practice. A quarter of the clinics used boiling water devices as the sterilizing instrument. However, in a study to evaluate the efficiency of boiling water, $81 \%$ of the microorganisms identified prior treatment remained viable. Because boiling may not disinfect adequately, doctors may be exposing their patients to the continuous risk of cross infection.

Disinfection: Majority of dentists in this study used betadine as their maior disinfectant. Betadine and 
phenol alcohol have been shown to provide a low level of disinfection. Chemical germicides, registered as a "hospital disinfectant" and labeled for "tuberculocidal" activity, are recommended for disinfecting surfaces that have been soiled with patient material. These intermediate-level disinfectants include phenolics, iodophors, and chlorine-containing compounds. Again, the current practice is not on par with the recommendation.

Handwashing: Although $99 \%$ of dentists reported using the gloves, only $90 \%$ washed their hands prior examination. Unwashed hands can contaminate gloves while they are being put on. Soap and water removes only transient microorganisms acquired directly or indirectly from patient contact; therefore, for many routine dental procedures, such as examinations and non-surgical techniques, hand-washing with plain soap though recommended is not sufficient.

Disposal: Disposal of potentially infectious wastes was frequently (45\%) included with the other general wastes. In addition to direct contamination, injuries from the needles and other sharps contaminated with blood or body fluids present a great risk of transmitting blood borne diseases. According to the standard precaution, used disposable syringes and needles, scalpel blades, and other sharps should be placed in appropriate puncture-resistant containers.

Vaccination: Only $85 \%$ of the dentists and $35 \%$ of the dental assistants were found to be vaccinated against the HBV. The US Occupational Safety and Health Administration requires that employers make hepatitis $B$ vaccinations available without cost to their employees who may be exposed to blood or other infectious materials . In addition, CDC (Centers for Disease Control and Prevention) recommends that all workers, including dental health care workers, who might be exposed to blood or blood-contaminated substances in an occupational setting, be vaccinated for HBV.

\section{Conclusions and recommendations}

The present study found that many dentists do not utilize the full range of recommended infection control procedures that are necessary to minimize the risk of cross-infection in dental practice. As the infectious status of the patient is often unknown, to prevent contamination and cross infection either to the patient or to the dentist, it is necessary to treat all patients as potentially infectious.

In the light of current findings, following recommendations are made:

1. The "standard precautions" regarding sterilization, disinfection, and hand washing should be followed strictly by all dental professionals and their implementation should be monitored by NMC.

2. There should be better policy guidelines regarding dental waste disposal, including sharps.

3. Provision of vaccine against blood borne pathogens, e.g. HBV, should be made mandatory for dental surgeons and other dental auxiliaries.

4. Dental colleges and training programmes for dental axillaries should devote more time in their curriculum to infectious disease control procedures.

5. Additional studies and monitoring are needed to assess the risk of transmission during dental procedures, as well as compliance with proper safe practice guidelines.

\section{Acknowledgement}

The Authors are greatly indebted to Dr.Freddrick A. Connell, Associate Dean, School of Public Health, University of Washington for his editorial assistance.

\section{References}

1. American Dental Association. Infection control for the Dental office and the Dental Laboratory -2002[Homepage on internet]. Chicago: American Dental Association; Available from: http/www.ada.org/prof/pubs/nifg.html

2. Ahtone J, Goodman RA. Hepatitis B and dental personnel: transmission to patients and prevention issues. J Am Dent Assoc. 1983; 106:219-22.

3. Ciesielski C, Marianos D, Chin-Yih OU, et al. Transmission of human immunodeficiency virus in a dental practice. Ann Intern Med. 1992; 116:798-5.

4. Dooley SW, Castro KG, Hutton MD, Mullan RJ, Polder JA, Snider DE. Guidelines for preventing the transmission of tuberculosis in health-care settings, with special focus on HIVrelated issues. MMWR. 1990 Dec 07 / 39(RR17);1-29.

5. Anonymous. Recommendations for preventing transmission of human immunodeficiency virus and hepatitis B virus during exposure-prone invasive procedures. MMWR. 1991 Jul 12 / 40(RR08);1-9.

6. Garner JS. Guideline for prevention of surgical wound infections, 1985. Atlanta: CDC, 1985; publication no. 99-2381.

7. Garner JS, Favero MS. Guideline for hand washing and hospital environmental control, 1985. Atlanta: CDC, 1985; publication no. 991117. 
8. Aizawa F,Yone MM, Hanada NA. Survey on infection control practices, knowledge and attitudes toward HIV AIDS among dental practioners. Nippon-Koshu-Eisei-zasshi. 1996 May; 43(5): 364-73.

9. CDC. Recommendations for prevention of HIV in health-care settings. MMWR. 1987 Aug 21;36(2S):3S-18S.
10. CDC. Guidelines for prevention of transmission of human immunodeficiency virus and hepatitis $B$ virus to health-care and public-safety workers. MMWR. 1989; 38(suppl. No. S-6):1-37.

11. CDC. Protection against viral hepatitis: recommendations of the Immunization Practices Advisory Committee (ACIP). MMWR 1990; 39(No. RR-2). 